\section{Increased failure rates after the introduction of the TFNA proximal femoral nail for trochanteric fractures: implant related or learning curve effect?}

\author{
Peter P SCHMITZ ${ }^{1}$, Gerjon HANNINK ${ }^{2}$, Joey REIJMER ${ }^{1}$, Matthijs P SOMFORD ${ }^{1}$, \\ and Job L C VAN SUSANTE 1
}

\author{
${ }^{1}$ Department of Orthopedics, Rijnstate Hospital, Arnhem; ${ }^{2}$ Department of Operating Rooms, Radboud University Medical \\ Center Nijmegen, Nijmegen, The Netherlands \\ Correspondence: jvansusante@rijnstate.nl \\ Submitted 2021-07-11. Accepted 2021-12-19.
}

Background and purpose - Trochanteric fractures are often treated using intramedullary fixation. In our institution, the TFN-Advanced Proximal Femoral Nailing System (TFNA) was introduced as replacement for the Gamma Trochanteric Nail (GTN3) for the treatment of these fractures as a result of a hospital-driven change of trauma implant supplier. We compared trochanteric fracture fixation failure rate between these 2 intramedullary nails.

Patients and methods - All trochanteric fractures treated surgically from 2011 to 2019 were retrospectively reviewed for fixation failure. From 2016 only the TFNA was used. Fixation failure was defined as implant cut-out, implant breakage, non-union, malpositioning of the screw/ blade requiring reoperation, new fracture around the nail, or miscellaneous. Propensity score matching was used to balance distribution of covariates and to compare failure rates between TFNA and GTN3 groups. Learning curve analyses were performed.

Results - After exclusion, 797 GTN3s (779 patients) and 542 (536 patients) TFNAs were available for analysis. A higher risk of fixation failure was found in the TFNA group (14\%) compared with the GTN3 group (7.0\%) (hazard ratio [HR] 2.0, 95\% confidence interval [CI] 1.2-3.5). This was mainly attributed to a higher risk of cut-out (HR 2.2; CI 0.95.7), malpositioning (HR 4.7; CI 0.7-34), and new fracture around the nail (HR 4.0; CI 1.0-16). Learning curve analyses indicated no clear learning curve effect.

Interpretation - Failure of fixation increased after a switch from the GTN3 to the TFNA proximal femoral nail for the treatment of trochanteric fractures. Cut-out and malpositioning of the calcar screw or blade appeared to be the most dominant failure mechanisms. Modifications in implant design may have played a role in this increased risk of failure of fixation. In our institution a new implant device was intro- duced without solid clinical evidence behind it. This study may help to underline the need for medical doctors with a critical and scientific background to be involved in implant choices.

Trochanteric fractures are mostly treated with intramedullary fixation. Failures of fixation are not uncommon (4-7\%) and lead to reoperation in most cases (1).

The third generation of the Gamma Trochanteric Nail (GTN3; Stryker, Mahwah, NJ, USA) is a widely used implant with established reliability and safety as documented in several clinical studies $(\mathbf{2}, \mathbf{3})$. In 2015, the TFN-Advanced Proximal Femoral Nailing System (TFNA) was introduced to the global market, claiming to potentially decrease fixation failure rates through a number of implant changes.

From 2016 the TFNA (DePuy Synthes, West Chester, PA, USA) was used in our institution as replacement for the GTN3 (Stryker) for the treatment of trochanteric fractures. This switch in implant found its origin in a hospital-driven change of trauma implant supplier. We now compare trochanteric fracture fixation failure rates between the 2 intramedullary nails, Gamma Trochanteric Nail (GTN3) and the TFN-Advanced Proximal Femoral Nailing System (TFNA) and evaluate the responsible underlying mechanisms of failure. Since the TFNA was introduced as a new device we also assessed a potential learning curve effect.

\section{Patients and methods}

Electronic files of all patients treated with an intramedullary nail following a trochanteric fracture between June 2011 and July 2019 were screened. Patients suffering from pathological 


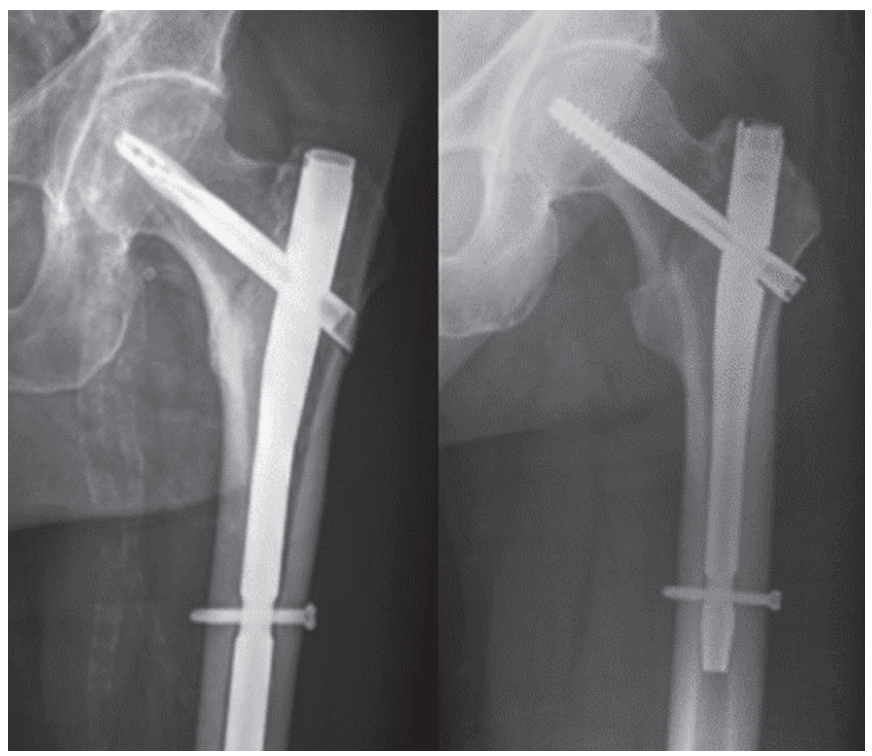

Figure 1. A TFN-Advanced Proximal Femoral Nail (TFNA; left) and a third-generation Gamma Trochanteric Nail (GTN3; right). Note the TFNA's implant modifications: the use of a blade instead of a screw, oblique cut on lateral end of the blade, different bowing, smaller proximal diameter, and different design of the proximal hole.

fractures were excluded. The TFNA was introduced to treat trochanteric fractures in our institution in 2016 whereas the GTN3 had been used since 2004. No GTN3-nail option was available after the introduction of the TFNA in 2016. Patient characteristics regarding age, sex, BMI, smoking status, and ASA score were collected. Length of stay and operation time were noted. Furthermore, type of fracture was defined according to the AO fracture classification system (4). As implant positioning may influence chances of failure of fixation (5), the tip-apex distance (TAD) (6) was assessed on intraoperative anterior-posterior and axial fluoroscopy as proxy measure for implant positioning.

\section{Outcomes}

This study assesses radiographic failure of fixation. 6 categories of failure were established and each case was allocated to 1 of the following: 1) implant cut-out, 2) implant breakage, 3 ) non-union, 4) malpositioning of the screw/blade in the femoral head, 5) new fracture around the nail, and 6) miscellaneous. An implant cut-out was defined as clear migration of hardware in the femoral head and implant breakage as a discontinuity in the device. Non-union was defined as radiographic absence of bone bridging at the fracture site for which reoperation had occurred to remove the distal locking screw. Malpositioning of the screw/blade in the femoral head was defined as an inadequate position requiring early re-intervention according to established consensus from the weekly trauma meeting. A new fracture around the nail was defined as the occurrence of a fracture related to a new fall. Lastly, a small remaining heterogeneous group of failures from collapse of the femoral head, either post-traumatic or from avascular necrosis, was classified as miscellaneous.

\section{Implants and surgery}

The TFNA and GTN3 are roughly similar implants (Figure 1) and are both available in long and short nails. The standard nail has a diameter of $11 \mathrm{~mm}$ for both implants and a length of $200 \mathrm{~mm}$ in the TFNA versus $180 \mathrm{~mm}$ in the GTN3. Both implants have the option for a femoral neck angle of 125 or 130 degrees. For both implants a calcar screw can be used, whereas for the TFNA this screw could now be replaced by a helical blade, which was 1 of the main modifications in implant design (Figure 1). This helical blade was designed to compress bone during insertion to enhance implant anchorage and to reduce the risk of cut-out (7). A blade was used within the TFNA in all patients above 70 years old. For both types of implants we used a similar operative technique. Patients were operated on in a supine position with the use of a traction table and fluoroscopy for closed reduction. All patients received preoperative cefazolin 2,000 mg and nadroparin $2850 \mathrm{IE}$ antiXa till 5 weeks postoperatively. Postoperatively, patients were encouraged to fully weight-bear. With an incidence of around 150 trochanteric fractures per year in our institution all surgeries were performed by or under direct supervision of a certified (orthopedic) trauma surgeon.

\section{Statistics}

As a crude analysis, Kaplan-Meier analysis was performed to determine the failure of fixation rates, stratified for implant type (GTN3 or TFNA).

Multiple imputation by chained equations was used to account for missing data. We generated 30 imputed datasets, as current guidance recommends that 1 imputation should be performed per percent of incomplete observations (8). ASA score, BMI, smoking status, and nail size had 2.6\%, 5.8\%, $30 \%$, and $0.3 \%$ missing values, respectively (Table 1 ).

To adjust for potential confounding baseline characteristics, we matched patients based on their propensity scores. The propensity score was defined as the probability of being treated with either TFNA or GTN3 dependent on a case's recorded baseline characteristics. Propensity scores were estimated for each imputed dataset, using a logistic regression model with the group (TFNA or GTN3) as the dependent variable in relation to the following baseline characteristics: age, sex, ASA score, BMI, smoking, fracture type, and length of nail (short or long). The selection of which variables to include in our analyses in order to minimize bias was done using directed acyclic graphs based on the approaches described by Shrier and Pearl (9,10). A 1:1 nearest-neighbor matching algorithm was applied without replacement to match cases on their corresponding propensity scores within a caliper of 0.2 standard deviations of the logit of the propensity score (11). The balance between the 2 groups after matching was checked graphically and descriptively. A standardized difference of less than 
Table 1. Patient characteristics and missing data among the TFNA and GTN3 groups from the unadjusted cohort. Values are n (\%) unless otherwise specified

\begin{tabular}{lcccc}
\hline & \multicolumn{2}{c}{ TFNA } & \multicolumn{2}{c}{ GTN3 } \\
Characteristic & $(n=542)$ & Missing & $(\mathrm{n}=797)$ & Missing \\
\hline Mean age (SD) & $79(14)$ & $0(0)$ & $80(12)$ & $0(0)$ \\
Female sex & $378(70)$ & $0(0)$ & $589(74)$ & $0(0)$ \\
ASA score & & $0(0)$ & & $37(4.6)$ \\
1 & $32(5.9)$ & N/A & $35(4.4)$ & N/A \\
2 & $134(25)$ & N/A & $315(40)$ & N/A \\
3 & $276(51)$ & N/A & $362(45)$ & N/A \\
4 & $90(17)$ & N/A & $37(4.6)$ & N/A \\
5 & $10(1.8)$ & N/A & $13(1.6)$ & N/A \\
Mean BMI (SD) & $24.1(4.4)$ & $21(4)$ & $24.0(4.2)$ & $56(7)$ \\
Smoking, yes & $90(17)$ & $0(0)$ & $112(14)$ & $398(50)$ \\
Fracture type & & $0(0)$ & & $0(0)$ \\
A1 & $206(38)$ & N/A & $290(36)$ & N/A \\
A2 & $234(43)$ & N/A & $326(41)$ & N/A \\
A3 & $80(15)$ & N/A & $131(16)$ & N/A \\
Subtrochanteric & $22(4.1)$ & N/A & $50(6.3)$ & N/A \\
Screw/blade & & $0(0)$ & & $0(0$ \\
Screw & $142(26)$ & & $797(100)$ & \\
Blade & $400(74)$ & $0(0)$ & N/A & $4(0.5)$ \\
Size & & & \\
Short & $419(77)$ & N/A & $620(78)$ & N/A \\
Long & $123(23)$ & N/A & $173(22)$ & N/A \\
Mean TAD (SD) & $19.8(6.8)$ & $28(5.2)$ & $17.6(6.1)$ & $41(5.1)$ \\
Days of stay (SD) & $8.8(6.1)$ & $0(0)$ & $9.6(7.1)$ & $0(0)$ \\
Operation time, & & & & \\
minutes (SD) & $45(22)$ & $0(0)$ & $44(23)$ & $0(0)$ \\
\hline
\end{tabular}

TFNA - TFN-Advanced Proximal Femoral Nailing System GTN3 - Third generation of the Gamma Trochanteric Nail. $\mathrm{N} / \mathrm{A}$, not applicable; TAD, tip-apex-distance in $\mathrm{mm}$.

$10 \%$ indicates an appropriate balance (11). Mean standardized mean differences (SMD) with their range across the 30 multiple imputed datasets are presented in Figure 2 and Table 2 (see Supplementary data).

On each of the 30 imputed and propensity score-matched datasets, we used Cox regression to analyze the effect of intramedullary nail on failure of fixation (12). The resulting hazard ratios (HRs) and absolute risk differences were pooled using Rubin's rule. Absolute risk differences were estimated at 3 years' follow-up. 95\% confidence intervals (CI) were calculated for the HRs and absolute risk differences.

As the TFNA was newly introduced in our institution, we used the exponential weighted moving average (EWMA) method to assess a potential learning curve. EWMA is a well-known statistical process monitoring tool because of its exceptional pace in catching infrequent variations in the process parameters, and is commonly used to monitor hospital data (13).

By the choice of weighting factor, $\lambda$, the EWMA procedure can be made sensitive to either detect a small change or a trend in the process. We used a $\lambda$ of 0.005 to discern a trend in the observed outcomes (14). We performed a learning curve analysis on overall fixation failures, operation (OR) time, cut-out, and malpositioning. Besides overall fixation failures, OR time

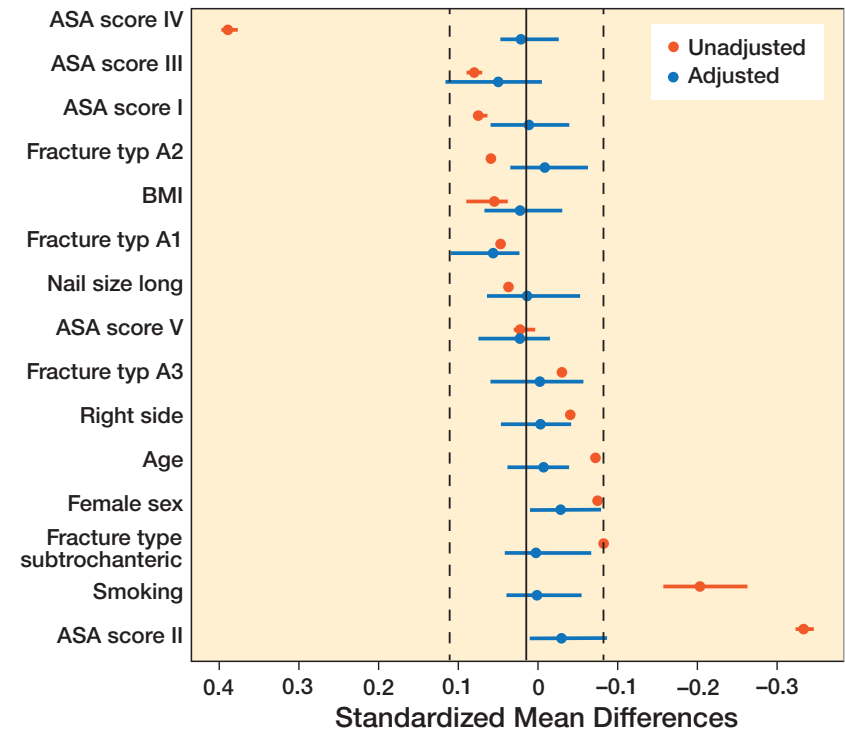

Figure 2. Love plot showing mean standardized mean differences (between TFNA and GTN3) with their range across the 30 multiple imputed datasets before (unadjusted) and after propensity-score matching (adjusted).

was chosen because a relatively prolonged operation time is anticipated on the introduction of a new implant. Both cut-out and malpositioning were chosen from the subcategories for a learning curve analysis as for these 2 in particular a potential learning curve effect can be anticipated. In addition, we performed sensitivity analyses excluding the first 50,150, and 250 TFNA cases to assess the influence of a potential learning curve on the results.

Statistical analyses were performed using R 3.6.3 (R Foundation for Statistical Computing, Vienna, Austria) using packages "mice," "cobalt," "MatchIt," and "riskRegression."

\section{Ethics, data sharing, funding, and potential conflicts of interest}

Ethical approval for the study was granted by the Institutional Review Board (decision 2020-1596). The datasets generated during and/or analyzed during the current study are available from the corresponding author on reasonable request. No funding was obtained and the authors have no conflicts of interest to declare.

\section{Results}

1,377 intramedullary nails were implanted in 1,353 consecutive patients. In 562 (556 patients) trochanteric fractures a TFNA was placed, and 815 (797 patients) trochanteric fractures were treated with a GTN3. After exclusion of 20 patients in the TFNA group and 18 patients in the GTN3 group due to a pathologic fracture, 542 (536 patients) TFNAs and 797 (779 patients) GTN3s were included in this study. Fixation failure rates for both groups are presented in Figure 3. 


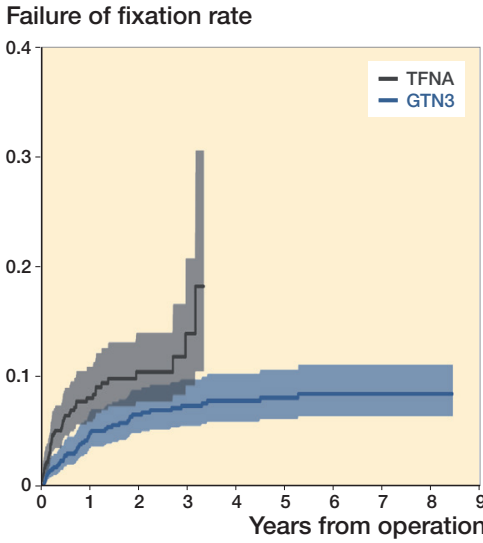

Figure 3. Cumulative incidence with $95 \% \mathrm{Cl}$ of failure of fixation for TFNA and GTN3. P-value $=0.002$ (log-rank test). For TFNA and GTN3 abbreviations, see Table 1.
Failure rate - any failure

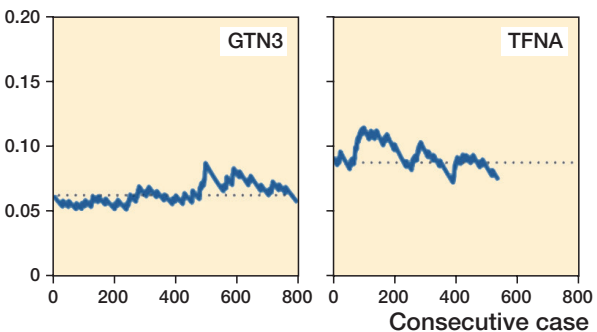

Consecutive case

Failure rate - malposition
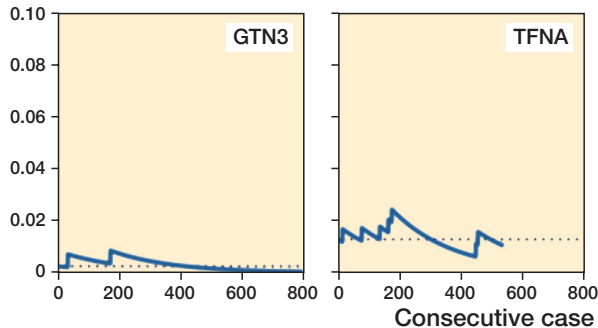

Failure rate - cut-out
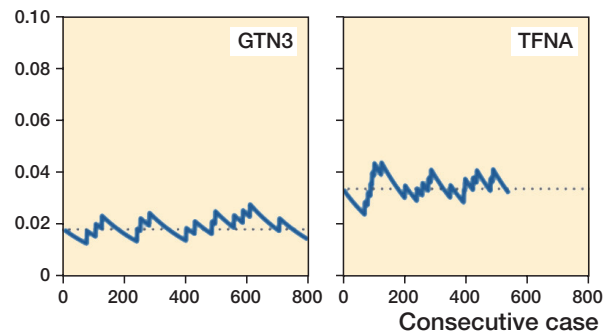

Operation time (min)

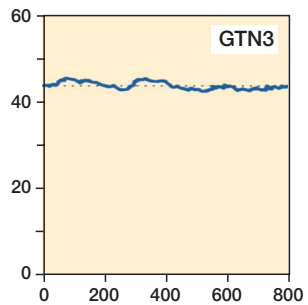

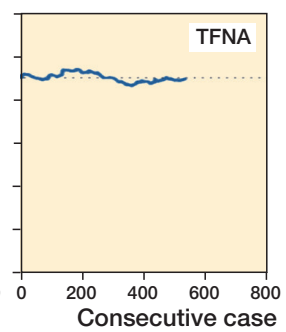

Figure 4. Exponentially weighted moving average (EWMA) plots for overall failures, implant cutout, malpositioning, and operation time for both TFNA and GTN3. Dotted lines illustrate the mean failure rates during the study period. For TFNA and GTN3 abbreviations, see Table 1.

\section{TFNA vs. GTN3 after propensity-score matching}

The Cox regression analysis revealed a failure of fixation of $14 \%$ in the TFNA group versus $7.0 \%$ in the GTN3 group, resulting in a hazard ratio (HR) of 2.0. The absolute risk difference of fixation failure in the TFNA group was $6.8 \%$ higher at 3 years' follow-up compared with the GTN3 group (Table 3).

The failures in the TFNA group versus the GTN3 group for each subgroup were: cut-out in $4.8 \%$ and $2.2 \%$, implant breakage in $0.6 \%$ and $0.6 \%$, non-union in $1.6 \%$ and $1.7 \%$, malpositioning in $1.5 \%$ and $0.3 \%$, new fracture around the nail in $5.2 \%$ and $1.4 \%$, and miscellaneous in $1.2 \%$ and $1.0 \%$ (Table 3 ).

\section{Learning curve}

The TFNA EWMA plots revealed fluctuating curves around the mean without a clear decreasing trend in time for all 4 outcomes, indicative of the absence of a clear learning curve effect in the TFNA group (Figure 4). A separate sensitivity

Table 3. Hazard ratios (HR) and estimated absolute risk differences at 3 years in each group

\begin{tabular}{llccc}
\hline Factor & HR $(95 \% \mathrm{Cl})$ & $\begin{array}{c}\text { TFNA } \\
(\%)\end{array}$ & $\begin{array}{c}\text { GTN3 } \\
(\%)\end{array}$ & $\begin{array}{c}\text { Risk difference } \\
(\%(95 \% \mathrm{Cl}))\end{array}$ \\
\hline Overall failure & $2.0(1.2-3.5)$ & 14 & 7.0 & $6.8(1.7$ to 12$)$ \\
Cut-out & $2.2(0.9-5.7)$ & 4.8 & 2.2 & $2.6(0.1$ to 5.2$)$ \\
Implant breakage & $1.1(0.1-9.0)$ & 0.6 & 0.6 & $0.0(-1.2$ to 1.2$)$ \\
Non-union & $0.9(0.3-3.3)$ & 1.6 & 1.7 & $-0.2(-2.4$ to 2.1$)$ \\
Malpositioning & $4.7(0.7-34)$ & 1.5 & 0.3 & $1.2(0.0$ to 2.5$)$ \\
Fracture around nail & $4.0(1.0-16)$ & 5.2 & 1.4 & $3.9(-0.8$ to 8.5$)$ \\
Miscellaneous & $1.2(0.2-8.2)$ & 1.2 & 1.0 & $0.1(-1.9$ to 2.2$)$ \\
\hline
\end{tabular}

For TFNA and GTN3 abbreviations, see Table 1. analysis excluding the first 50,150, and 250 TFNA cases showed our results for fixation failure of the TFNA versus the GTN3 to be robust (Table 4, see Supplementary data).

\section{Discussion}

This study revealed an increased risk of fixation failure in the TFNA group. We found a hazard ratio (HR) of 2.0 (CI 1.2$3.5)$ for fixation failure in the TFNA group compared with the GTN3 group. Cut-out and malpositioning appeared to be the most commonly encountered failure mechanisms. Learning curve analyses revealed that the potential influence of a learning curve could only have been minimal.

The TFNA was introduced to the global market with a number of innovative implant modifications to improve clinical performance. To date, literature regarding the TFNA is limited to a few small case series reporting fixation failure rates of 2-7\% (15-18). Amongst these case series, 2 recent studies also reported concerns about mechanically failed TFNA implants $(17,18)$.

The higher risk of fixation failure in the TFNA group we found was mainly attributed to a higher risk of cut-out, malpositioning, and new fracture around the nail. In particular, the failure mechanisms cut-out and malpositioning may have been influenced by surgical technique. As learning curve analyses revealed no clear learning effect the encountered increase in fixation failures appears to be at least partly implant related. Several different features were introduced at a time within the TFNA such as a different anatomical fit, a smaller proximal diameter (LATERAL RELIEF CUT 
design), an oblique cut on the lateral end of the blade/screw, and a different design of the proximal hole (BUMP CUT design) (7). Lastly, the blade has been introduced to replace the calcar screw. Biomechanical studies presented in the manufacturer's value analysis brief reported a significantly higher resistance to cut-out of the TFNA helical blade compared with the TFNA screw $(7,19)$.

2 clinical studies using the TFNA reported on higher rates of implant cut-out with the use of a helical blade compared with lag-screw fixation in the treatment of trochanteric fractures with intramedullary devices $(\mathbf{2 0 , 2 1 )}$. These reports are in line with our study where $4.8 \%$ fixation failure was encountered in the TFNA group from implant cut-out versus $2.2 \%$ in the GTN3 group (Table 3). Of note is that a blade was used in $74 \%$ of our cases in the TFNA group because an age cut-off above 70 years old was used for the blade. As such, one may argue whether this concept of a blade versus a screw is indeed an improvement. In fact, the blade is hammered into the femoral head and so fracture distraction is introduced whereas compression is commonly accepted to increase chances of fracture healing. Furthermore, the oblique cut on the lateral end of the blade/screw was introduced to reduce lateral protrusion on the soft tissues (7). One could argue that the higher risk of malpositioning encountered in the TFNA group may partly have been the consequence of this innovation as the blade/screw can now only be inserted in the femoral head with steps of 360 degrees of rotation to align the lateral cortex while before more subtle 90-degree steps of insertion were an option.

Our study has limitations. Due to the retrospective design, confounding is likely to be present. In an attempt to minimize this potential confounding, we performed propensityscore matching of patients on important confounding factors. Despite matching of patients on a subset of baseline characteristics, differences in unmeasured covariates probably still exist. For example, both an inter- and intra-surgeon difference concerning indication for reoperation cannot be ignored. This potential bias appears to be limited, as indication for reoperation was established by consensus between all orthopedic/ trauma surgeons in weekly trauma meetings throughout the entire study period, thus avoiding single-surgeon decisions. Nevertheless, a randomized controlled trial is the ideal tool to control for bias. Furthermore, relatively small absolute differences are found in fixation failure rates between groups, and one may argue whether these differences are clinically relevant. Nonetheless, modifications in implant designs are aimed to improve clinical results and as such a decrease in clinical outcome, irrespective of a small margin, does not match with true gain in sustainability. Lastly, we did not correct for correlated bilateral cases in the analysis, while the methods of our statistical analysis do assume independent observations. However, previous studies showed bilateral surgeries do not introduce dependency problems in register studies $(\mathbf{2 2 , 2 3 )}$.

From the limited data available in the literature on TFNA together with the encountered relatively high risk of fixation failure in this study there is reason to raise questions concerning the clinical benefit of the modifications applied to this device. In general, there is a tendency to overestimate the benefit of a newly introduced device (24). Newly introduced implants are often embraced by professionals after strong marketing without solid evidence (25). This rapid uptake of newly introduced implants into the market is, however, not without risks, in particular patients' health-related risks. For this reason, a structured introduction and regulated clinical monitoring of implant innovations has repeatedly been recommended (25). Such regulation and monitoring may also apply to trauma devices for internal fixation. In hindsight, we regret that also in our institution a new implant device was introduced without solid clinical evidence behind it. An institution-driven larger change of (trauma) implant supplier was the driving force behind this. This study may help to underline the need for medical doctors with a critical and scientific background to be at least involved in implant choices.

In conclusion, this study revealed an increased risk of fixation failure in the TFNA proximal femoral nail in comparison with the Gamma nail (GTN3) for the treatment of trochanteric fractures. Cut-out and malpositioning were the most commonly encountered failure mechanisms. Modifications in implant design may have played a role in this increased risk of failure of fixation, because no clear learning curve effect was found.

PS and JS designed the study; PS and JR collected the data; GH carried out data analyses; PS, GH, and JS wrote the manuscript; PS, GH, MS, and JS contributed to revision of the manuscript.

Acta thanks Charles Court-Brown and Per Kjærsgaard-Andersen for help with peer review of this study.

1. Adams C I, Robinson C M, Court-Brown C M, McQueen M M. Prospective randomized controlled trial of an intramedullary nail versus dynamic screw and plate for intertrochanteric fractures of the femur. J Orthop Trauma 2001; 15(6): 394-400. doi: 10.1097/00005131200108000-00003.

2. Bonnaire F, Lein T, Fülling T, Bula P. Reduced complication rates for unstable trochanteric fractures managed with third-generation nails: Gamma 3 nail versus PFNA. Eur J Trauma Emerg Surg 2020; 46(5): 95562. doi: 10.1007/s00068-019-01200-7.

3. Pascarella R, Fantasia R, Maresca A, Bettuzzi C, Amendola L, Violini S, et al. How evolution of the nailing system improves results and reduces orthopedic complications: more than 2000 cases of trochanteric fractures treated with the Gamma Nail System. Musculoskelet Surg 2016; 100(1): 1-8. doi: 10.1007/s12306-015-0391-y.

4. Marsh J L, Slongo T F, Agel J, Broderick J S, Creevey W, DeCoster T A, et al. Fracture and dislocation classification compendium-2007: Orthopaedic Trauma Association classification, database and outcomes committee. J Orthop Trauma 2007; 21(10 Suppl.): S1-133. doi: 10.1097/00005131-200711101-00001.

5. Hsueh K K, Fang C K, Chen C M, Su Y P, Wu H F, Chiu F Y. Risk factors in cutout of sliding hip screw in intertrochanteric fractures: an evaluation of 937 patients. Int Orthop 2010; 34(8): 1273-6. doi: 10.1007/ s00264-009-0866-2. 
6. Baumgaertner M R, Curtin S L, Lindskog D M, Keggi J M. The value of the tip-apex distance in predicting failure of fixation of peritrochanteric fractures of the hip. J Bone Joint Surg Am 1995; 77(7): 1058-64. doi: 10.2106/00004623-199507000-00012.

7. Synthes D.TFN-Advanced Proximal Femoral Nailing System: value analysis brief 2017. Available from: http://synthes.vo.llnwd.net/o16/LLNWMB8/ INT\%20Mobile/Synthes\%20International/Product\%20Support\%20Material/legacy_Synthes_PDF/DSEM-TRM-0515-0375-1_LR.pdf.

8. Van Buuren S. Flexible imputation of missing data. 2nd ed. Boca Raton, FL: CRC Press; 2018.

9. Shrier I, Platt R W. Reducing bias through directed acyclic graphs. BMC Med Res Methodol 2008; 8: 70. doi: 10.1186/1471-2288-8-70.

10. Pearl J. Understanding propensity scores. Causality: models, reasoning, and inference Cambridge: Cambridge University Press; 2010. p. 348-52.

11. Austin P C. Optimal caliper widths for propensity-score matching when estimating differences in means and differences in proportions in observational studies. Pharm Stat 2011; 10(2): 150-61. doi: 10.1002/pst.433.

12. Sayers A, Evans J T, Whitehouse M R, Blom A W. Are competing risks models appropriate to describe implant failure? Acta Orthop 2018; 89(3): 256-8. doi: 10.1080/17453674.2018.1444876.

13. Morton A, Mengersen K, Playford G, Whitby M. Statistical methods for hospital monitoring with R. Chichester UK: Wiley; 2013.

14. Cook D A, Coory M, Webster R A. Exponentially weighted moving average charts to compare observed and expected values for monitoring risk-adjusted hospital indicators. BMJ Qual Saf 2011; 20(6): 469-74. doi: 10.1136/bmjqs.2008.031831.

15. Yee D K H, Lau W, Tiu K L, Leung F, Fang E, Pineda J P S, et al. Cementation: for better or worse? Interim results of a multi-centre cohort study using a fenestrated spiral blade cephalomedullary device for pertrochanteric fractures in the elderly. Arch Orthop Trauma Surg 2020. doi: 10.1007/s00402-020-03449-9.

16. Matsumura T, Takahashi T, Nakashima M, Nibe Y, Takeshita K. Clinical outcome of mid-length proximal femoral nail for patients with trochanteric hip fractures: preliminary investigation in a Japanese cohort of patients more than 70 years old. Geriatr Orthop Surg Rehabil 2020; 11 : 2151459320936444. doi: 10.1177/2151459320936444.
17. Lambers A, Rieger B, Kop A, D'Alessandro P, Yates P. Implant fracture analysis of the TFNA proximal femoral nail. J Bone Joint Surg Am 2019; 101(9): 804-11. doi: 10.2106/jbjs.18.00997.

18. Klima M. Comparison of early fatigue failure of the TFNa and Gamma 3 cephalomedullary nails in the United States from 20152019. J Orthop Trauma 2021; 35(2): e329-344. doi: 10.1097/ bot.0000000000001864.

19. Hofmann L. Final report for biomechanical evaluation of non-augmented nail head elements in surrogate femoral heads [Synthes $\mathrm{GmbH}$ : USTRA09022 Trochanteric Fixation Nail - Advanced (TFNA)] 2015.

20. Stern L C, Gorczyca J T, Kates S, Ketz J, Soles G, Humphrey C A. Radiographic review of helical blade versus lag screw fixation for cephalomedullary nailing of low-energy peritrochanteric femur fractures: there is a difference in cutout. J Orthop Trauma 2017; 31(6): 305-10. doi: 10.1097/bot.0000000000000853.

21. Chapman T, Zmistowski B, Krieg J, Stake S, Jones C M, Levicoff E. Helical blade versus screw fixation in the treatment of hip fractures with cephalomedullary devices: incidence of failure and atypical "medial cutout". J Orthop Trauma 2018; 32(8): 397-402. doi: 10.1097/ bot.0000000000001193.

22. Robertsson $\mathbf{O}$, Ranstam J. No bias of ignored bilaterality when analysing the revision risk of knee prostheses: analysis of a population based sample of 44,590 patients with 55,298 knee prostheses from the national Swedish Knee Arthroplasty Register. BMC Musculoskelet Disord 2003; 4: 1. doi: 10.1186/1471-2474-4-1.

23. Park M S, Kim S J, Chung C Y, Choi I H, Lee S H, Lee K M. Statistical consideration for bilateral cases in orthopaedic research. J Bone Joint Surg Am 2010; 92(8): 1732-7. doi: 10.2106/jbjs.I.00724.

24. Parker M J, Cawley S. Short (175 mm) versus standard (220 mm) length intramedullary nail for trochanteric hip fractures: a randomized trial of 229 patients. Bone Joint J 2020; 102-b(3): 394-9. doi: 10.1302/0301620x.102b3.Bjj-2019-0776.R3.

25. van Susante J L C. The (r)evolution of hyped innovations in orthopedic implants: can prudent introduction avoid throwing the baby out with the bathwater? Acta Orthop 2019; 90(6): 507-10. doi: 10.1080/17453674.2019.1669115 


\section{Supplementary data}

Table 2. Balance across multiple imputations before and after propensity score matching (PSM)

\begin{tabular}{|c|c|c|c|c|c|c|}
\hline \multirow[b]{2}{*}{ Characteristic } & \multicolumn{3}{|c|}{ Balance across imputations before PSM } & \multicolumn{3}{|c|}{ Balance across imputations after PSM } \\
\hline & TFNA & GTN3 & Mean SMD (range) & TFNA & GTN3 & Mean SMD (range) \\
\hline Mean age (SD) & $78.8(13.5)$ & $79.9(11.8)$ & $-0.09(-0.09$ to -0.09$)$ & $79.0(12.7)$ & $79.2(12.7)$ & $-0.02(-0.06$ to 0.02$)$ \\
\hline \multicolumn{7}{|l|}{ ASA score, $\%$} \\
\hline 1 & 5.9 & 4.5 & 0.06 (0.05 to 0.07$)$ & 5.6 & 5.8 & $-0.003(-0.06$ to 0.05$)$ \\
\hline 2 & 25 & 41 & $-0.36(-0.37$ to -0.35$)$ & 28 & 30 & $-0.04(-0.10$ to -0.01$)$ \\
\hline 3 & 51 & 45 & 0.068 (0.06 to 0.08$)$ & 56 & 54 & $0.04(-0.02$ to 0.10$)$ \\
\hline 4 & 17 & 4.6 & $0.39(0.37$ to 0.40$)$ & 8.0 & 7.8 & $0.01(-0.04$ to 0.03$)$ \\
\hline 5 & 1.8 & 1.6 & 0.01 ( -0.01 to 0.02$)$ & 2.1 & 2.0 & $0.010(-0.03$ to 0.06$)$ \\
\hline Mean BMI (SD) & $24.1(4.4)$ & $24.0(4.2)$ & $0.04(0.02$ to 0.08$)$ & $24.1(4.4)$ & $24.1(4.3)$ & $0.01(-0.05$ to 0.05$)$ \\
\hline \multirow{2}{*}{\multicolumn{7}{|c|}{ Fracture type, \% }} \\
\hline & & & & & & \\
\hline A1 & 38 & 36 & $0.03(0.03$ to 0.03$)$ & 40 & 38 & $0.04(-0.01$ to 0.01$)$ \\
\hline A2 & 43 & 41 & 0.05 (0.05 to 0.05$)$ & 40 & 41 & $-0.02(-0.08$ to 0.02$)$ \\
\hline A3 & 15 & 16 & $-0.05(-0.05$ to -0.05$)$ & 15 & 16 & $-0.02(-0.07$ to 0.05$)$ \\
\hline Subtrochanteric & 4.1 & 6 & $-0.10(-0.10$ to -0.10$)$ & 4.6 & 4.7 & $-0.01(-0.08$ to 0.03$)$ \\
\hline \multicolumn{7}{|l|}{ Size, $\%$} \\
\hline Short & 77 & 78 & $0.023(0.02$ to 0.02$)$ & 78 & 78 & $-0.001(-0.07$ to 0.05$)$ \\
\hline Long & 23 & 22 & & 22 & 22 & \\
\hline
\end{tabular}

Balance between groups is presented as mean standardized mean differences (SMD) with the corresponding minimum and maximum standardized mean differences across the 30 multiple imputed datasets. Patient characteristics are presented as pooled mean (SD) for continuous variables or as pooled percentages for binary or categorical variables across multiple imputations. For TFNA and GTN3 abbreviations, see Table 1.

Table 4. Hazard ratios of fixation failure of the TFNA with scenarios excluding different numbers of TFNA cases to elucidate a potential learning curve effect

\begin{tabular}{ll}
\hline Scenario & HR $(95 \% \mathrm{Cl})$ \\
\hline All cases & $2.0(1.2-3.3)$ \\
First 50 TFNA cases excluded & $2.1(1.1-3.7)$ \\
First 150 TFNA cases excluded & $1.6(0.8-3.0)$ \\
First 250 TFNA cases excluded & $2.4(1.0-5.9)$ \\
\hline
\end{tabular}

TFNA = TFN-Advanced Proximal Femoral Nailing System. 